\title{
Individual Attributes Influencing Cervical Cancer Screening Services in Selected Health Facilities in Machakos County
}

\author{
Agnes Wavinya Nzioka ${ }^{1, *}$, Akunga Daniel Nyagetiria ${ }^{2}$, Wanyoro Anthony Karanja ${ }^{3}$ \\ ${ }^{1}$ School of Public Health, Kenyatta University, Nairobi, Kenya \\ ${ }^{2}$ Department of Environmental Health, Kenyatta University, Nairobi, Kenya \\ ${ }^{3}$ Department of Obstetrics and Gynecology, Kenyatta University, Nairobi, Kenya
}

Email address:

agnesnzioka2012@gmail.com(A.W.Nzioka)

${ }^{*}$ Corresponding author

\section{To cite this article:}

Agnes Wavinya Nzioka, Akunga Daniel Nyagetiria, Wanyoro Anthony Karanja. Individual Attributes Influencing Cervical Cancer Screening Services in Selected Health Facilities in Machakos County. Journal of Cancer Treatment and Research. Vol. 6, No. 1, 2018 , pp. 1-6. doi: $10.11648 /$ j.jctr.20180601.11

Received: March 7, 2018; Accepted: April 8, 2018; Published: May 7, 2018

\begin{abstract}
In Kenya, 51.2\% of estimated women population of 11 million aged 15 years and older are at risk of developing cervical cancer. Research shows that screening a woman even once between the ages of 35 and 40 years reduces her lifetime risk of cervical cancer by $25-36 \%$. However, the effective cervical cancer screening in developing countries is as low as 18.5\%. Low levels of utilization of Cervical Cancer Screening Services (CCSS) among health care workers have been documented. This study sought to determine the utilization of cervical cancer screening services by healthcare workers (HCWs) in selected health facilities in Machakos County. It was also thought imperative to determine individual $\mathrm{HCW}$ attributes influencing utilization of cervical cancer screening services in selected health care facilities in the county. The study adopted a descriptive cross-sectional study design involving 271 female health workers drawn from all the level 4 and level 5 facilities within the county. Respondents were stratified according to level of facility and according to their cadres. Data was collected via self-administered questionnaires and a facility assessment tool. Data analysis used SPSS version 21. Association was subjected to binary logistic regression. Only $25 \%$ of HCWs had utilized cervical cancer screening services. HCWs who had certificate $(\mathrm{OR}=0.05$, $\mathrm{p}$-value $<0.01)$, diploma $(\mathrm{OR}=0.04$, $\mathrm{p}$-value $<0.01)$ and degrees $(\mathrm{OR}=0.09$, $\mathrm{p}$-value $=0.01)$ were less likely to utilize CCSS as compared to HWCs who had attained graduate studies or higher. Also health workers aged 30 years and below $(\mathrm{OR}=0.12$, $\mathrm{p}$-value $=0.01)$ and those aged between 31 and 40 years $(\mathrm{OR}=0.30, \mathrm{p}$-value $=0.05)$ were less likely to utilize CCSS as compared to healthcare workers who were aged more than 50 years. The study recommends establishment of tailor-made sensitization programs on young female health care worker aimed at increasing their uptake of CCSS services among these group.
\end{abstract}

Keywords: Cervical Cancer, Cervical Cancer Screening Services, Female Healthcare Workers, Individual Attributes

\section{Introduction}

\subsection{Background Information}

Cervical cancer (CC) is a slow-onset malignancy found in the interior lining of the cervix, at the junction of the vagina and uterus [1]. CC is has been majorly linked to high- risk HPV types 16 and 18, early sexual debut, high parity, multiple sexual partners and co-infection with HIV [2]. Others include Chlamydia trachomatis, herpes simplex virus type 2, immunosuppressant, diet, smoking [3, 4] This infection results in transformation of the cervical epithelial cells first, to precancerous lesions and then to frank cancer [5]. Worldwide CC, is the fourth most common cancer affecting women after breast, colorectal and lung cancers and the second common reproductive tract cancer among women [6].

In sub-Saharan Africa, 34.8 new cases of cervical cancer 
per 100,000 women are diagnosed yearly and 22.5 per 100,000 women diagnosed with cervical cancer die annually [7]. In Kenya cervical cancer is not only the second cause of reproductive tract cancer among women but also the leading cause of cancer deaths among women aged between 15 and 49 years [8]. It is estimated there are 4,802 CC cases annually and causes 2,451deaths annually in Kenya [9]. The National Cervical Cancer Prevention Strategic Plan (NCCPSP) seeks to ensure that Women of Reproductive Age (WRA) have access to cervical cancer prevention and control services thereby reducing the incidence of overt cancer with its impact on health and development [8].

$\mathrm{CC}$ is preventable and its morbidity and mortality could be greatly reduced using preventative health methods such as HPV vaccination and safe sexual practice and most importantly secondary prevention through screening [5]. Cervical cancer screening is estimated to prevent up to $80 \%$ of CC in countries where screening is routine [10]. Research has demonstrated that screening a woman even once between the ages of 35 and 40 years reduces her lifetime risk of cervical cancer by $25-36 \%$ [11]. Furthermore screening enables early detection and treatment of pre-malignant lesions and referral for cervical cancer management which will improve length and quality of life of the health care workers, besides being affordable and cost effective [11].

In limited resource settings WHO-approved, strategy for cervical cancer screening in low resource settings is visual inspection with acetic acid (VIA) or visual inspection with Lugol's iodine (VILI) [10]. Pap smear is the most commonly used method for cervical cancer screening and is only available in urban areas but these facilities experience frequent shortages of materials needed for taking Pap smears [12]. Screening for cancer of the cervix recommended for all sexually active females [13]. Therefore health workers are expected to act as role models in uptake of preventive services like cervical cancer screening [14].

\subsection{Problem Statement}

Worldwide, low level of coverage in cervical cancer screening services and wide inequalities in the utilization of those services has been reported $[15,7]$. Crude coverage of cervical cancer screening in developed countries is at $93.6 \%$ while in developing countries the crude coverage is only $44.7 \%$ [15].

However, previous studies in several countries have documented low levels of utilization of cervical cancer screening services among health care workers [4, 16, 14]. Worrisomely, health care workers like the general population continue to be diagnosed with cervical cancer when it is too late when little on nothing can be done, or when the burden for treatment is too high to manage $[17,18,19]$. In Kenya, uptake of cervical cancer screening services is low, at $3.5 \%$ [9]. Furthermore, individual factors that influence effective utilization of the standardized screening services among health care workers was not adequately documented either in Kenya or in selected health facilities in Machakos County, hence the need for this study.

\subsection{Objectives}

1. To establish the proportion of health care workers who utilize cervical cancer screening services in selected health facilities in Machakos County

2. To determine individual factors influence on utilization of cervical cancer screening services among the health care workers in selected health facilities in Machakos County.

\section{Methodology}

This was a descriptive cross sectional research that utilized quantitative research method of data collection and analysis conducted in 4 level 4 and 1 level 5 public health facilities among female health workers. A minimum sample of 251 female health workers was targeted, however 271 health workers responded to the study instruments. Stratified sampling was used to sample health workers proportionate to number of health workers in each level and professional cadres. Data was collected through face-to-face interviews using structured questionnaires.

Authority to carry out the study was sought from NACOSTI. Approval to conduct research was sought from Kenyatta University Ethical Review Committee; and permission to collect data was sought from the authorities in each hospital. Respondents consented to participate with confirmation of confidentiality. Collected data was crosschecked for completeness and any missing entries corrected upon completion of each interview.

Data coding, entry and analysis was done using the SPSS version 20 software. The questionnaires were inspected to detect errors and omissions and corrected immediately. Multiple binary logistic regression used to test the association between CCSS utilization and individual characteristics of health workers. Findings were presented as text, graphs and tables.

\section{Findings and Discussion}

\subsection{CCSS Utilization}

Only a quarter $69(25 \%)$ of the respondents had previously utilized CCSS services while $203(75 \%)$ had never utilized CCSS. This shows that CCSS utilization in the county among health workers were relatively higher as compared to screening prevalence in developing countries which is $18.5 \%$ $[15,7]$. However, this proportion was significantly higher compared to the general uptake of CCSS in Kenya which is estimated to be as low as $3.2 \%$ [9]. This was also similar to other previous studies in several countries that documented low levels of utilization of cervical cancer screening services among health care workers [14, 4, 16, 19]. But this was still low since health workers are expected to be role models in developing awareness, confidence and compliance of women for CCSS especially if they have been screened themselves [20-22]. 


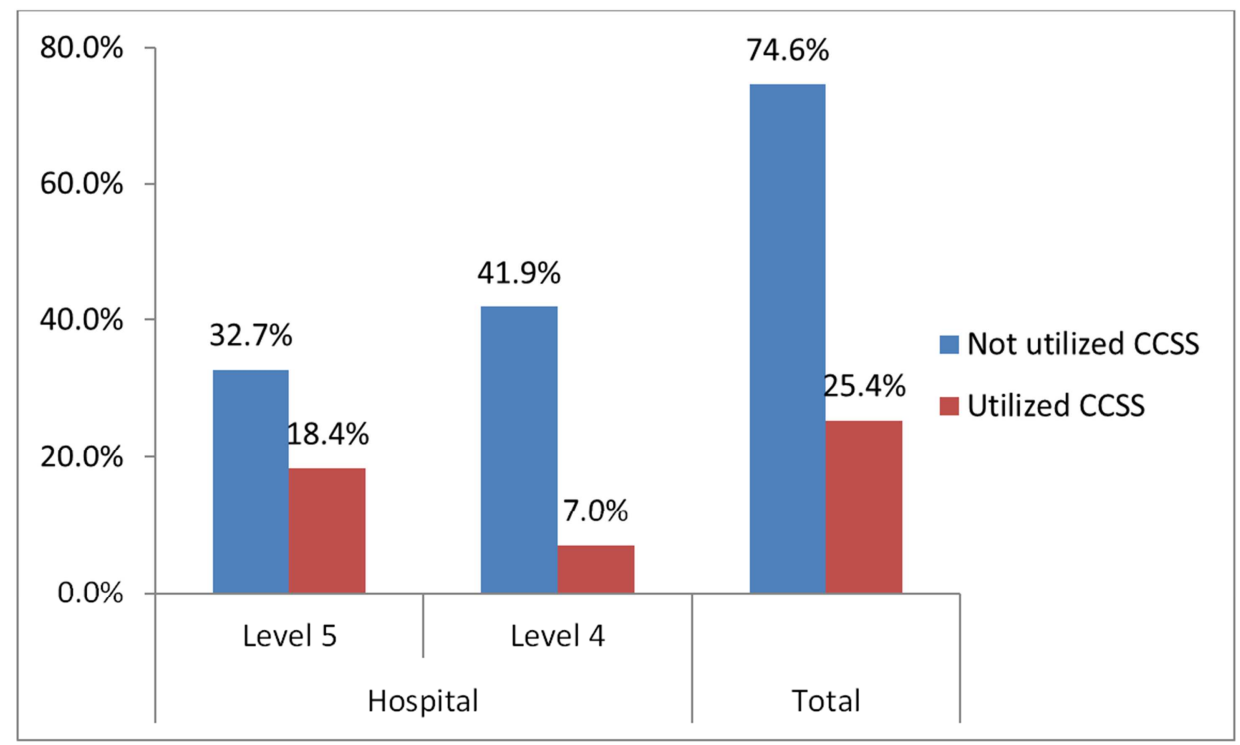

Figure 1. CCSS Utilization.

\subsection{Socio-Demographic Characteristics and CCSS Utilization}

Health workers who had certificate (Adjusted Odds Ratio $[\mathrm{AOR}]=0.05, \mathrm{p}$-value $<0.01)$, diploma $(\mathrm{AOR}=0.04$, $\mathrm{p}$ value $<0.01)$ and degree $(\mathrm{AOR}=0.09, \mathrm{p}$-value $=0.01)$ were less likely to utilize CCSS compared to HCWs who had attained postgraduate studies. This showed that an increase in education level results to an increase in CCSS utilization. This finding was similar to a study in Cameroon where access to CCSS was found to be determined by women's level of education which effectively determined the level of knowledge on cervical cancer and its prevention [27]. Contrary observation was made also among HCWs in Ethiopia which showed that there were no significant difference in CCSS utilization among HCWs with diploma and those with degrees [19].

HCWs aged 30 years and below $(\mathrm{OR}=0.108, \mathrm{p}$-value $=$ 0.009 ) and those aged between 31 and 40 years were less likely to utilize CCSS as compared to nurses who were aged more than 50 years. Health workers aged above 50 years had the highest proportion $(47.6 \%)$ of CCSS utilization. This showed that older HCWs were more likely to utilize CCSS as compared to younger ones. This could be due to the fact that elderly women may receive more frequent gynecological care and the common belief that cervical cancer only occurs in older women, making them more responsive to their health and young HCWs not seeing themselves as susceptible [14]. Similar findings were made in Nigeria [24-26].

Table 1. CCSS Utilization and HCWs Socio-Demographic Characteristics.

\begin{tabular}{|c|c|c|c|c|c|c|c|}
\hline \multirow{2}{*}{ Characteristic } & \multirow{2}{*}{ Categories } & \multicolumn{2}{|c|}{ Not utilized CCSS } & \multicolumn{2}{|c|}{ Utilized CCSS } & \multirow{2}{*}{ AOR $(95 \%$ CI) } & \multirow{2}{*}{ p-value } \\
\hline & & $\mathbf{F}$ & $\%$ & $\mathbf{F}$ & $\%$ & & \\
\hline \multirow{3}{*}{ Marital status } & Married & 142 & 71.7 & 56 & 28.3 & $1.35(0.55-3.30)$ & 0.52 \\
\hline & Divorced & 7 & 87.5 & 1 & 12.5 & $0.12(0.01-1.68)$ & 0.12 \\
\hline & Never married (Ref) & 54 & 81.8 & 12 & 18.2 & & \\
\hline \multirow{2}{*}{ Profession } & Clinical & 136 & 72.7 & 51 & 27.3 & $0.79(0.39-1.61)$ & 0.52 \\
\hline & Non-clinical (Ref) & 67 & 78.8 & 18 & 21.2 & & \\
\hline \multirow{4}{*}{ Education level } & Certificate & 33 & 66.0 & 17 & 34.0 & $0.05(0.01-0.32)$ & 0.00 \\
\hline & Diploma & 139 & 79.4 & 36 & 20.6 & $0.04(0.01-0.25)$ & 0.00 \\
\hline & Degree & 28 & 75.7 & 9 & 24.3 & $0.09(0.01-0.59)$ & 0.01 \\
\hline & Postgraduate (Ref) & 3 & 30.0 & 7 & 70.0 & & \\
\hline \multirow{3}{*}{ Number of children } & 0 & 38 & 79.2 & 10 & 20.8 & $1.64(0.48-5.62)$ & 0.43 \\
\hline & $1-2$ & 108 & 80.6 & 26 & 19.4 & $0.82(0.39-1.71)$ & 0.60 \\
\hline & $3-5$ (Ref) & 57 & 63.3 & 33 & 36.7 & & \\
\hline \multirow{4}{*}{ Age } & $<30$ & 74 & 90.2 & 8 & 9.8 & $0.12(0.02-0.64)$ & 0.01 \\
\hline & $31-40$ & 61 & 81.3 & 14 & 18.7 & $0.30(0.09-1.00)$ & 0.05 \\
\hline & $41-50$ & 46 & 63.0 & 27 & 37.0 & $0.58(0.25-1.36)$ & 0.21 \\
\hline & $>50$ (Ref) & 22 & 52.4 & 20 & 47.6 & & \\
\hline \multirow{3}{*}{ Work experience } & $<10$ & 102 & 88.7 & 13 & 11.3 & $0.50(0.13-1.91)$ & 0.31 \\
\hline & $10-20$ & 57 & 70.4 & 24 & 29.6 & $0.88(0.36-2.14)$ & 0.78 \\
\hline & $>20$ (Ref) & 44 & 57.9 & 32 & 42.1 & & \\
\hline
\end{tabular}




\subsection{CCSS Knowledge and CCSS Utilization}

To measure knowledge levels of individual respondents, a series of eight dichotomous true/false questions were asked. Each respondent who answered a question rightly was deemed knowledgeable and was awarded a score of one and zero otherwise. In the end these scores were added to indicate CCSS knowledge scores with a maximum score of 8 points and minimum 0 points. The results were then rated as high knowledge level ( $\geq 7$ points), moderate ( $5-6$ points) and low ( $\leq 4$ points). 191 (70.2\%) of the participants were moderately knowledgeable, $57(21.0 \%)$ were highly knowledgeable while $24(8.8 \%)$ had low knowledge. There was no significant association between CCSS knowledge and CCSS utilization among HCWs in the County. This finding disagrees with a study in Tanzania which revealed that knowledge of cervical cancer and its prevention was a barrier to CCSS among health care workers [3]. Literature indicates that that lack of relevant and timely knowledge about cervical cancer in the population and among health care workers is a prime barrier for access to cervical cancer prevention [23]. However, previous studies among female health workers, show that health workers were knowledgeable about several health related issues but this knowledge had not translated to better uptake of health services among these health workers $[21,16,22,26]$.

Table 2. CCSS Knowledge and CCSS Utilization.

\begin{tabular}{|c|c|c|c|c|c|}
\hline \multirow{2}{*}{ CCSS knowledge } & \multicolumn{2}{|c|}{ CCSS utilization } & \multirow{2}{*}{ Total $(N=272)$} & \multirow{2}{*}{$\operatorname{AOR}(95 \%$ CI) } & \multirow{2}{*}{ p-value } \\
\hline & Not utilized & Utilized & & & \\
\hline Low & $16(66.7 \%)$ & $8(33.3 \%)$ & $24(8.8 \%)$ & $1.54(0.54-4.35)$ & 0.419 \\
\hline Moderate & $144(75.4 \%)$ & $47(24.6 \%)$ & $191(70.2 \%)$ & $1.0(0.50-1.99)$ & 0.994 \\
\hline High (Ref) & $43(75.4 \%)$ & $14(24.6 \%)$ & $57(21.0 \%)$ & & \\
\hline
\end{tabular}

\subsection{CCSS Attitude and CCSS Utilization}

Perception and attitude towards CCSS was also measured using series of five 5-point Likert scale questions spanning from strongly agree to strongly disagree which were later merged to a binomial agree and disagree items; and further a score was then developed such that positive response was awarded a point while negative response was not which were then summed up to indicate positive, negative or neutral attitude. In the end these scores were added to indicate the CCSS perception and attitude with a maximum score of 5 points and minimum 0 points. The results were then rated as positive attitude ( $\geq 4$ points), neutral $(2-3$ points) and negative attitude ( $\leq 1$ point). 217 (79.8\%) HCWs had positive attitude, $36(13.2 \%)$ were neutral while $19(7.0 \%)$ had negative attitude. There was no significant association between CCSS attitude and CCSS utilization among HCWs. Contrary observation was made among HCWs in Ethiopia which showed that negative attitudes among HCWs deterred them from utilizing CCSS [19]. Contrary findings were also made among female health workers in Nigeria who were found to have negative attitude towards cervical cancer screening which contributed to low CCSS utilization [27]. These findings however agrees with a study in Nigeria HCWs had good attitude of nurses towards Pap smear but did not did not translate to better utilization [26].

Table 3. CCSS Attitude and CCSS Utilization.

\begin{tabular}{lllll}
\hline \multirow{2}{*}{ CCSS attitude } & CCSS utilization & & \multirow{2}{*}{ Total (N = 272) } & \multirow{2}{*}{ AOR (95\% CI) } \\
\cline { 2 - 3 } & Not utilized & Utilized & $19(7.0 \%)$ & $1.03(0.35-2.98)$ \\
Negative & $14(73.7 \%)$ & $5(26.3 \%)$ & $36(13.2 \%)$ & $.82(0.35-1.91)$ \\
Neutral & $28(77.8 \%)$ & $8(22.2 \%)$ & $217(79.8 \%)$ & 0.961 \\
Positive & $161(74.2 \%)$ & $56(25.8 \%)$ & 0.647 & \\
\hline
\end{tabular}

\section{Conclusions}

1. There was low utilization of CCSS services among health workers in Machakos County with only a quarter of healthcare workers having ever been screened. This was better in level 5 hospital as compared to level 4 hospitals but much better than the national CCSS utilization.

2. Healthcare workers who had certificate and diploma were less likely to utilize CCSS as compared to healthcare workers who had attained postgraduate studies healthcare workers. Also healthcare workers aged 30 years and below were less likely to utilize CCSS as compared to healthcare workers who were

aged more than 50 years.

\section{Recommendations}

1. The findings show that only a quarter of HCWs in the selected health facilities in Machakos County had utilized CCSS. Therefore there is need for more sensitization among HCWs and service providers to increase uptake of available CCSS for them to function as champions not only for the HCWs but also for the general public.

2. It has been shown that utilization was low among HCWs below 30 years; therefore, there is need for the Machakos County to enhance early sensitization and support programs among young female healthcare 
workers aimed at increasing their uptake of the available CCSS.

\section{Acknowledgements}

The authors of this publication would like to thank Elijah Kipchumba for data management and analysis services.

\section{Competing Interests}

All authors declare that: there are no significant competing financial, professional or personal interests that might have influenced the performance or presentation of the work described in this manuscript.

\section{Conflict of Interest}

All authors report no conflicts of interest in this publication.

\section{Authors' Contributions}

Authors made substantial contributions to conception and design, and/or acquisition of data, and/or analysis and interpretation of data.

\section{References}

[1] Robert A. Smith et al., "Cancer screening in the United States, 2015: A review of current American Cancer Society guidelines and current issues in cancer screening," CA: A Cancer Journal for Clinicians, vol. 65, no. 1, pp. 30-54, 2015, doi:10.3322/caac. 21261 .

[2] Jean Damascène Makuza et al., "Prevalence and risk factors for cervical cancer and pre-cancerous lesions in Rwanda," The Pan African Medical Journal, vol. 22, no. 26, 2015, doi: 10.11604/pamj.2015.22.26.7116.

[3] Frida S Lyimo and Tanya N Beran, "Demographic, knowledge, attitudinal, and accessibility factors associated with uptake of cervical cancer screening among women in a rural district of Tanzania: Three public policy implications," BMC Public Health, vol. 12, no. 22, 2012, doi: 10.1186/14712458-12-22.

[4] I. O. G. Owoeye and I. A Ibrahim, "Knowledge and attitude towards cervical cancer screening among female students and staff in a tertiary institution in the Niger Delta," International Journal of Medicine and Biomedical Research, vol. 2, no. 1, pp. 48-56, 2013, http://www.ajol.info/index.php/ijmbr/article/view/91935/8822 5 .

[5] Hyacinth I. Hyacinth, Oluwatoyosi A. Adekeye, Joy N. Ibeh, and Tolulope Osoba, "Cervical Cancer and Pap Smear Awareness and Utilization of Pap Smear Test among Federal Civil Servants in North Central Nigeria," PLoS One, vol. 7, no. 10, p. e46583, 2012, doi: 10.1371/journal.pone.0046583.

[6] Freddie Bray, Jian-Song Ren, Eric Masuyer, and Jacques Ferlay, "lobal estimates of cancer prevalence for 27 sites in the adult population in 2008," International Journal of Cancer, vol. 132, no. 5, pp. 1133-1145, 2013, http://onlinelibrary.wiley.com/doi/10.1002/ijc.27711/pdf.

[7] International Agency for Research on Cancer (IARC). (2013) The International Agency for Research on Cancer (IARC). [Online]. https://www.iarc.fr/en/mediacentre/pr/2013/pdfs/pr223_E.pdf.

[8] Ministry of Medical Services \& Ministry of Public Health and Sanitation, The National Cervical Cancer Prevention Strategic Plan (2012 -2015). Nairobi: Ministry of Medical Services \& Ministry of Public Health and Sanitation, 2012.

[9] L Bruni et al., "Human Papillomavirus and Related Diseases in Kenya," Barcelona, Spain, Summary Report 2016-02-26 2016.

[10] Sarah Finocchario-Kessler et al., "Cervical cancer prevention and treatment research in Africa: a systematic review from a public health perspective," BMC Women's Health, vol. 16, no. 29, pp. 1-25, 2016, DOI: 10.1186/s12905-016-0306-6.

[11] Sue J. Goldie et al., "Cost-Effectiveness of Cervical-Cancer Screening in Five Developing Countries," The New England Journal of Medicine, vol. 353, pp. 2158-2168, 2005, DOI: 10.1056/NEJMsa044278.

[12] Lucy W Kivuti-Bitok, Ganesh P Pokhariyal, Roudsari Abdul, and Geoff McDonnell, "An exploration of opportunities and challenges facing cervical cancer managers in Kenya," BMC Research Notes, vol. 6, no. 136, pp. 1-10, 2013, doi: 10.1186/1756-0500-6-136.

[13] Prudence Creighton et al., "Cervical cancer screening in Australia: modelled evaluation of the impact of changing the recommended interval from two to three years," BMC Public Health, vol. 10, no. 374, pp. 1-14, 2010, DOI: 10.1186/14712458-10-734.

[14] Oyedunni Sola Arulogun and Opemipo Olubunmi Maxwell, "Perception and utilization of cervical cancer screening services among female nurses in University College Hospital, Ibadan, Nigeria," The Pan African Medical Journal, vol. 11, no. 69 , pp. $1-8,2012$,

http://www.ncbi.nlm.nih.gov/pmc/articles/PMC3361207/.

[15] Emmanuela Gakidou, Stella Nordhagen, and Ziad Obermeyer, "Coverage of Cervical Cancer Screening in 57 Countries: Low Average Levels and Large Inequalities," PLoS Medicine, vol. 5, no. 6, p. e132, 2008, doi: 10.1371/journal.pmed.0050132.

[16] Alok Goyal, Gunvant Vaishnav, Anjani Shrivastava, Ragini Verma, and Anjali Modi, "Knowledge, attitude \& practices about cervical cancer and screening among nursing staff in a teaching hospital," International Journal of Medical Science and Public Health, vol. 2, no. 2, pp. 249-253, 2013, http://www.scopemed.org/?mno=30507.

[17] Etienne Asonganyi et al., "Factors Affecting Compliance with Clinical Practice Guidelines for Pap Smear Screening among Healthcare Providers in Africa: Systematic Review and MetaSummary of 2045 Individuals," PLoS ONE, vol. 8, no. 9, p. e72712, 2013, doi:10.1371/journal.pone.0072712.

[18] Innocent O. Maranga et al., "Analysis of Factors Contributing to the Low Survival of Cervical Cancer Patients Undergoing Radiotherapy in Kenya," PLoS One, vol. 8, no. 10, p. e78411, 2013, doi: 10.1371/journal.pone.0078411. 
[19] Mihret Gebreegziabher, Nigus Gebremedhin Asefa, and Semarya Berhe, "Factors Affecting the Practices of Cervical Cancer Screening among Female Nurses at Public Health Institutions in Mekelle Town, Northern Ethiopia, 2014: A Cross-Sectional Study," Journal of Cancer Research, vol. 2016, no. 2016, p. Article ID 4743075, 2016, http://dx.doi.org/10.1155/2016/4743075.

[20] Adenike O Akhigbe and Vivian O Omuemu, "Knowledge, attitudes and practice of breast cancer screening among female health workers in a Nigerian urban city," BMC Cancer, vol. 9, no. 203, pp. 1-9, 2009, DOI: 10.1186/1471-2407-9-203.

[21] Rudolph Kantum Adageba, Kwabena Antwi Danso, Frank Kokroe Ankobea, David Zawumya Kolbilla, and Paul Opoku, "Knowledge of cervical cancer and patronage of cervical cancer screening services among female health workers in Kumasi, Ghana," Afr J Haematol Oncol, vol. 2, no. 1, pp. 157$161,2011$.

[22] V Shah, S Vyas, A Singh, and M Shrivastava, "Awareness and knowledge of cervical cancer and its prevention among the nursing staff of a tertiary health institute in Ahmedabad, Gujarat, India," Ecancermedicalscience, vol. 6, no. 270, 2012, doi: 10.3332/ecancer.2012.270.

[23] Catherine McCarey et al., "Awareness of HPV and cervical cancer prevention among Cameroonian healthcare workers," BMC Women's Health, vol. 11, no. 45, 2011, DOI: $10.1186 / 1472-6874-11-45$.
[24] M. O. Oche, A. U. Kaoje, G Gana, and J. T. Ango, "Cancer of the cervix and cervical screening: Current knowledge, attitude and practices of female health workers in Sokoto, Nigeria," International Journal of Medicine and Medical Sciences, vol. 5, no. 4, pp. 184-190, 2013, http://www.academicjournals.org/article/article1378995320_O che $\% 20$ et $\% 20$ al.pdf.

[25] Maisaratu Bakari, Idris Usman Takai, and Mohammed Bukar, "Awareness and utilization of Papanicoloau smear among health care workers in Maiduguri, Nigeria," Niger J Basic Clin Sci, vol. 12, no. 1, pp. 34-38, 2015.

[26] O. Awodele et al., "A Study on Cervical Cancer Screening Amongst Nurses in Lagos University Teaching Hospital, Lagos, Nigeria," Journal of Cancer Education, vol. 26, no. 3, pp. 497-504, 2011, doi: 10.1007/s13187-010-0187-6.

[27] I. O Ehiemere, Maureen Dike Frank, and G. C RobinsonBassey, "Attitude and practice of cervical cancer screening among female health workers in university of Port-Harcourt teaching hospital, Rivers State," Journal of Research in Nursing and Midwifery, vol. 4, no. 5, pp. 072-082, 2015, http:/dx.doi.org/10.14303/JRNM.2015.0125. 